\title{
Tachyon Mediated Non-Gaussianity
}

\author{
Bhaskar Dutta* and Louis Leblond \\ Department of Physics, Texas A\&M University, College Station, TX 77843, USA \\ Jason Kuman \\ Department of Physics and Astronomy, University of California, Irvine, CA 92697, USA
}

\begin{abstract}
We describe a general scenario where primordial non-Gaussian curvature perturbations are generated in models with extra scalar fields. The extra scalars communicate to the inflaton sector mainly through the tachyonic (waterfall) field condensing at the end of hybrid inflation. These models can yield significant non-Gaussianity of the local shape, and both signs of the bispectrum can be obtained. These models have cosmic strings and a nearly flat power spectrum, which together have been recently shown to be a good fit to WMAP data. We illustrate with a model of inflation inspired from intersecting brane models.

PACS numbers: 98.80.Cq, 98.80.Es, 11.25.-w
\end{abstract}

Introduction. Single field slow-roll inflation generically predicts a near flat Gaussian spectrum of fluctuations in the curvature of spacetime. Gaussianity is a consequence of the slow-rolling conditions which require the inflaton to behave like a free field with negligible selfinteractions. We can parameterize the non-Gaussianity $(\mathrm{NG})$ in the curvature perturbation (denoted $\zeta$ here) using the so called $f_{N L}$ formalism:

$$
\zeta(\vec{x}, t)=\zeta_{\text {Gauss }}+\frac{3}{5} f_{N L}\left(\zeta_{\text {Gauss }}^{2}-\left\langle\zeta_{\text {Gauss }}^{2}\right\rangle\right),
$$

where $\zeta_{\text {Gauss }}$ is the Gaussian piece of the curvature perturbation. This ansatz for the curvature perturbations leads to the so-called local shape for the bispectrum in Fourier space.

The recent five year analysis from Wilkinson Microwave Anisotropy Probe (WMAP5) [1] shows $-9<$ $f_{N L}<111$ while Yadav and Wandelt (using WMAP3 data) have claimed a detection of primordial NG at above 99.5\% confidence level [2]. It is expected that the upcoming Planck experiment should be able to detect $f_{N L}$ of order unity. In the light of all these new and future measurements, it is very important to investigate the origin of large non-Gaussianity in models of inflation. It is also important to search for predictions that are associated with a larger $f_{N L}$ for other future observations, e.g., the shape of the bispectrum, running of $f_{N L}$, etc. The best bound on $f_{N L}$ given above is for the local shape. Another well known shape, the equilateral shape, has also been constrained (using WMAP3 data) to be $-256<f_{N L}^{\text {equil }}<332$ [3] while many other shapes have not yet been searched for individually.

For single field smooth slow-roll models with canonical kinetic terms, $f_{N L}$ is of order of the slow-roll parameters and is less than 1 [4]. In this paper, we investigate a natural way to induce large non-Gaussianity from a ubiquitous feature of string theory and models of physics beyond the standard model: extra scalars. In the following, we will refer to the fluctuation along the inflationary tra- jectory the adiabatic mode while orthogonal fluctuations are entropy modes. The entropy modes fluctuations are generically non-Gaussian since in general their potential is not flat. Given a mechanism to feed the entropy modes fluctuations into curvature perturbations, the curvature spectrum will also be non-Gaussian. In addition to this intrinsic NG of the entropy modes, the non-linearity of the transfer mechanism between entropy modes and curvature perturbations will also generate significant NG.

The scenario we present is general but can be easily realized by intersecting brane models (IBMs) in string theory [5]. The IBMs are well-motivated since in these scenarios not only the standard model can be constructed, but also the fermion mass hierarchies can be understood. From the effective field theory point of view, this scenario gives a multi-fields generalization of D-term inflation [6]. We will show that non-Gaussianity can be generated at the end of inflation in this type of models from two main different sources. The first source of NG is an "intrinsic" component coming from self interactions of the entropy modes. The second source is coming from the non-linear terms that arises when one transfers the entropy perturbations into curvature perturbations.

The intrinsic component of non-Gaussianity in our work is similar to the ref. [7] (building on previous work [8]) which also considers D-term inflation. The non-linear local piece on the other hand has been studied previously for multiple field hybrid inflation in [9] or in the context of modulated reheating [10]. But we find that in more general models, both contributions can appear and can be significant. Moreover, we argue that loop-contributions to the bispectrum can also be important. Thus, even simple, well-motivated models can yield a much more complicated picture than previously discussed.

The intrinsic and non-linear non-Gaussian contributions both have local shape with opposite signs that depend on the hidden-sector charge of the extra fields and on whether the dynamics of the entropy mode is dominated by the gauge sector or the Yukawa sector of the 
theory. Thus, we find in this setup that the sign of $f_{N L}$ is not a stochastic variable but rather fixed by the underlying physics of the system. We find that the best regime to obtain large NG $(10 \sim 100)$ is the regime of D-term inflation where we produce cosmic strings with tension $G \mu \sim 10^{-7}$ and where $n_{s} \sim 1$. This is a good fit to the WMAP data as recently shown in [11].

The 2-pt Correlation Function. In this work, we will be interested in a model of hybrid inflation containing the usual inflaton $\phi$ and the tachyonic (waterfall) field $T$ but extended with an extra scalar field $\chi$. During inflation, the tachyon has a positive mass and its VEV is pinned at zero. Below some critical value $\left(\phi_{c}\right)$ of the inflaton, the mass of the tachyon becomes negative and it condenses, ending inflation. We will assume that the $\chi$ field has low mass, fluctuates during inflation but does not directly couple to the inflaton field. Instead its only effect is to modulate the mass of the tachyon and to change the critical value at which inflation ends. In some ways, the tachyon acts as a messenger between the hidden sector field $\chi$ and the inflaton field $\phi$. The action for this system is of the following form

$$
\begin{aligned}
S & =\frac{1}{2} \int \sqrt{g}\left[M_{p}^{2} R-(\partial \phi)^{2}-(\partial T)^{2}-(\partial \chi)^{2}-2 V\right] \\
V & \left.=V_{\text {inf }}(\phi)+V_{\text {hid }}(\chi)+V_{\text {mess }}(\phi, \chi, T)\right]
\end{aligned}
$$

During inflation, the tachyonic field has vanishing VEV and the messenger part of the potential $V_{\text {mess }}$ vanishes as well (all coupling in this part of the potential involves the field $T$ ). Assuming that the hidden sector potential contribute a negligible amount to the vaccuum energy, the Hubble scale during inflation is equal to $H^{2}=\frac{V_{\text {inf }}(\phi)}{3 M_{p}^{2}}$.

This model is of the form considered in [7, 9]. Using the $\delta N$ formalism [12], we can compute the curvature perturbation $\zeta(\vec{x}, t)=\delta N$ where $N$ is the number of efolds. Since the inflationary potential is only a function of $\phi$, the field $\chi$ only affects the critical value at which inflation ends. The fluctuation in the number of efolds when $\phi \rightarrow \phi+\delta \phi$ and $\chi \rightarrow \chi+\delta \chi$ is

$$
\begin{aligned}
\delta N= & -\left.\frac{H}{\dot{\phi}} \delta \phi\right|_{*}+\left.\frac{H}{\dot{\phi}} \frac{\partial \phi_{c}}{\partial \chi} \delta \chi\right|_{\phi_{c}} \\
& +\left.\frac{1}{2} \frac{H}{\dot{\phi}} \frac{\partial^{2} \phi_{c}}{\partial \chi^{2}}\left(\delta \chi^{2}-\left\langle\delta \chi^{2}\right\rangle\right)\right|_{\phi_{c}}+\cdots,
\end{aligned}
$$

where the subscript $*$ means evaluated at horizon crossing. We have kept only the leading term at second order and we substracted a number to ensure that $\langle\delta N\rangle=0$. We will find that non-Gaussianities are generated both at linear order in the variable $\delta \chi$ as well as from the quadratic term. In curvaton models [13] (or more recently in ekpyrotic models [14]), NG is generated from these second order terms. The validity of the $\delta N$ expansion to higher orders and the NG that are generated from these terms were first discussed in [15]. We define

$$
\left.\gamma \equiv \frac{\partial \phi_{c}}{\partial \chi}\right|_{\phi_{c}}
$$

and this function encodes the coupling between $\chi$ and $\phi$ through the tachyonic field. To evaluate the 2-pt function we use the standard result that $\langle\delta \phi \delta \phi\rangle \sim H^{2} / 4 \pi^{2}$. Note that both $\delta \phi$ and $\delta \chi$ are not exactly constant outside of the horizon and they will decay exponentially because of their mass terms with $\left.\delta \chi\right|_{f} \sim \frac{H_{*}}{2 \pi} \kappa$ where

$$
\kappa=e^{-\int_{t_{*}}^{t_{f}} \eta_{\chi} H d t} \sim e^{-\eta_{\chi} N_{e}},
$$

and we used the following definitions for the slow-roll parameters.

$$
\epsilon=-\frac{\dot{H}}{H^{2}}, \quad \eta_{\varphi}=\frac{V_{, \varphi \varphi}}{3 H^{2}}, \quad \eta_{\chi}=\frac{V_{, \chi \chi}}{3 H^{2}} .
$$

Since we need to evaluate $\delta \chi$ at the end of inflation, significant non-Gaussianity will only arise if this damping is not too large, requiring $\eta_{\chi}$ to be small. However, $V_{\text {, }}$ need not be particularly small, which distinguishes $\chi$ from the inflaton. The 2-pt curvature correlation function is therefore [7]

$$
\begin{aligned}
\left\langle\zeta\left(\vec{k}_{1}\right) \zeta\left(\vec{k}_{2}\right)\right\rangle & =(2 \pi)^{3} \delta^{3}\left(\vec{k}_{1}+\vec{k}_{2}\right) 2 \pi^{2} \mathcal{P}_{2}^{\zeta} k^{-3}, \\
\mathcal{P}_{2}^{\zeta} & =\frac{H_{*}^{2}}{8 \pi^{2} M_{p l}^{2}}\left(\frac{1}{\epsilon_{*}}+\frac{\gamma^{2} \kappa^{2}}{\epsilon_{f}}\right),
\end{aligned}
$$

where $f$ means evaluated at the end of inflation. In the last expression, we have not included loop contributions coming from the higher order terms in the $\delta N$. They can be made small as we will discuss in more detail later on. The spectral index is easily computed from $n_{s}-1=$ $\frac{d \ln \mathcal{P}_{2}^{\zeta}}{d \ln k}$, the formula simplifies in the limit where $\epsilon_{*} \approx \epsilon_{f}$,

$n_{s}-1=\frac{1}{1+\gamma^{2} \kappa^{2}}\left(-6 \epsilon_{*}+2 \eta_{\varphi}+\gamma^{2} \kappa^{2}\left(-2 \epsilon_{*}+2 \eta_{\chi}\right)\right)$.

3-pt Correlation Function. The 3-pt correlation function can also be calculated using the same formalism although in this case we can neglect the part coming from the inflaton $\phi$ since it is going to be suppressed by power of the slow-roll parameters [4]. The field $\chi$ on the other hand can be non-Gaussian for a general potential $V_{\text {hid }}(\chi)$, and this non-Gaussianity can be transferred to the curvature spectrum. We will denote by $\left\langle\zeta^{3}\right\rangle_{\text {int }}$ the piece of the bispectrum which is intrinsic to the field $\delta \chi$ and arises directly from its higher-order self-interactions. This nonGaussianity in $\delta \chi$ is transferred to the $\delta N$ spectrum by the linear terms in eq. (3), yielding

$$
\left\langle\zeta^{3}\right\rangle_{\mathrm{int}}=\left.\left(\frac{H}{\dot{\phi}}\right)^{3} \gamma^{3}\left\langle\delta \chi^{3}\right\rangle\right|_{f} .
$$


One can calculate this term using the in-in formalism [4] with an interaction Hamiltonian simply given by $H_{I}=$ $\int d^{3} \vec{x} \sqrt{-g} V_{\text {hid }}(\chi)$. This computation was first done in [16] (see also 77, 17] for more recent derivations). The result is:

$$
\begin{aligned}
& \left\langle\zeta_{\vec{k}_{1}} \zeta_{\vec{k}_{2}} \zeta_{\vec{k}_{3}}\right\rangle_{\text {int }}=(2 \pi)^{3} \delta^{3}\left(\sum \vec{k}_{i}\right) \frac{H^{2} V_{, \chi \chi \chi} \kappa^{6}}{8 \sqrt{2} M_{p l}^{3} \epsilon_{f}^{\frac{3}{2}} \prod k_{i}^{3}} I_{3} \\
& I_{3}=\frac{4}{9} k_{t}^{3}-\frac{1}{3} \sum k_{i}^{3}\left(\gamma_{e}-N_{e}\right)-\sum_{i<j} k_{i} k_{j} k_{t}
\end{aligned}
$$

where $\gamma_{e}$ is the Euler number and we have defined $k_{t}=\sum k_{i}$. The number of efolds is $N_{e}=-\log \left(\frac{k_{t}}{a_{f} H_{f}}\right)$ and we have used the fact that in the slow-roll regime $\frac{H}{\dot{\phi}}=-\frac{1}{\sqrt{2 \epsilon} M_{p}}$. Since the number of efolds from horizon crossing is around 60 from observations, we see that the dominant term is $I_{3} \sim \frac{N_{e}}{3} \sum k_{i}^{3}$. This is exactly the local shape obtained from Eq. (11) (neglecting the weak momentum dependence in $N_{e}$ ). Indeed, Fourier transforming $\zeta$ in (1), one can compute the local 3-pt function to be

$$
\left\langle\zeta_{k}^{3}\right\rangle=(2 \pi)^{7} \delta^{3}\left(\sum \vec{k}_{i}\right) \frac{3}{10} f_{N L}\left(\mathcal{P}_{2}^{\zeta}\right)^{2} \frac{\sum k_{i}^{3}}{\prod k_{i}^{3}} .
$$

Comparing the last two expressions we find the following intrinsic contribution to the $f_{N L}$.

$$
f_{N L}^{\text {int }}=\frac{5}{9 \sqrt{2}} \frac{N_{e} M_{p} \gamma^{3} \kappa^{6}}{H^{2} \epsilon_{f}^{3 / 2}}\left(\frac{1}{\epsilon_{*}}+\frac{\gamma^{2} \kappa^{2}}{\epsilon_{f}}\right)^{-2} V_{, \chi \chi \chi} .
$$

where we use the subscript "int" to remind the reader that this NG is coming from self-interactions of the entropy modes. This kind of intrinsic NG occurs in models of inflation with non-trivial kinetic terms and they usually have very distinctive shapes in momentum space (see for example [18, 19]). In our case, the 3-pt function is dominated by the term proportional to the number of efolds which originates from the classical evolution of the field outside of the horizon and it therefore has the local shape. We should note that this type of intrinsic NG (with local shape) also occurs in model of non-local inflation where similar self-interactions are important [20].

In addition to the intrinsic NG, the non linear terms in Eq. (3) will also contribute to the bispectrum. In this case, Gaussian fluctuations in $\delta \chi$ are transferred nonlinearly to $\delta N$, inducing non-Gaussianity. Because the non-linear terms in the $\delta N$ expansion are local in spacetime, they will give a contribution of the local form and the leading term is

$$
f_{N L}^{\text {loc }}=-\frac{\partial \gamma}{\partial \chi} \frac{5 \gamma^{2} \kappa^{4} M_{p}}{3 \sqrt{2} \epsilon_{f}^{3 / 2}}\left(\frac{1}{\epsilon_{*}}+\frac{\gamma^{2} \kappa^{2}}{\epsilon_{f}}\right)^{-2}+\cdots
$$

where we have neglected higher order terms in the $\delta N$ expansion. Note that these two contributions have the same shape but opposite signs. The ratio of the intrinsic contribution to $f_{N L}$ compared to the local contribution is

$$
\beta \equiv\left|\frac{f_{N L}^{\mathrm{int}}}{f_{N L}^{\text {loc }}}\right|=\frac{1}{3} \frac{\gamma}{\gamma, \chi} \frac{V_{, \chi \chi \chi}}{H^{2}} N_{e} \kappa^{2}
$$

For the case where $\gamma$ is a constant (as in [7]), $f_{N L}^{\text {loc }}$ vanishes and the intrinsic contribution is the most important one. For the case where $\gamma$ is a linear function of $\chi$, we have $\gamma, \chi \sim \gamma / \chi$ and the ratio can be simplified to

$$
\beta \sim \eta_{\chi} N_{e} \kappa^{2}
$$

up to an order 1 coefficient which depends on the exact form of the potential. Given that one must have $\eta_{\chi}<$ $N_{e}^{-1}$ in order for the damping (c. f. Eq. (5) ) to be small, this ratio is usually smaller than 1 although for values of $\eta_{\chi} \sim 0.01$, it can be of order 1 .

Finally there are higher orders (loop) contributions to the 2-pt and 3-pt that we have not included here. For example, from Eq (3), there will be a correction to the 2-pt curvature function of the following form

$$
\left\langle\zeta_{k}^{2}\right\rangle_{\text {loop }} \supset\left(\frac{H}{\dot{\phi}}\right)^{2} \gamma, \chi \gamma\left\langle\delta \chi_{k} \int \frac{d^{3} k^{\prime}}{(2 \pi)^{3}} \delta \chi_{k-k^{\prime}} \delta \chi_{k^{\prime}}\right\rangle .
$$

This is a loop factor and the integral is IR divergent with a log that must be regulated. A natural cutoff is to use a box of the size of the universe today [21]. Then the loop factor is of order $\frac{\ln (k L)}{2 \pi^{2}} \sim \frac{1}{4}$ and using the intrinsic 3-pt function of $\delta \chi$ computed previously, we get a term of the order of

$$
-\frac{H_{*}^{2}}{8 \pi^{2} \epsilon_{f} M_{p l}^{2}} \gamma, \chi \gamma N_{e} \kappa^{6} V_{, \chi \chi \chi} \frac{\ln (k L)}{2 \pi^{2}} .
$$

For a given model, it is possible for this term to dominate over the $\gamma^{2}$ term in Eq. (7) although for the perturbation theory to remain under control, one should require that $\gamma$ is small enough to insure that $\delta \phi^{2}$ is the dominant term in the 2-pt. In addition one can get an additional contribution to the 2 -pt proportional to

$$
\left(\frac{H}{\dot{\phi}}\right)^{2} \gamma_{, \chi}^{2} \int \frac{d^{3} k^{\prime} d^{3} k^{\prime \prime}}{(2 \pi)^{6}}\left\langle\delta \chi_{k-k^{\prime}} \delta \chi_{k^{\prime}} \delta \chi_{k-k^{\prime \prime}} \delta \chi_{k^{\prime \prime}}\right\rangle
$$

This would give a contribution proportional to the square of the 2-pt function (of the order of $\frac{H^{4}}{\epsilon_{f} M_{p}^{2}} \gamma_{, \chi}^{2} \kappa^{4} \ln k L$ ) as well as a possible 2-loop contribution proportional to the 4-pt function. If we assume that $\gamma, \chi \chi$ is very small, we can stop here as all subsequent terms would be negligible. A systematic approach to calculate all these loops contributions has been developed recently in [22]. Similar terms contribute to the 3 -pt function as well [23]. A more thorough analysis of these additional contributions is important and we leave a detailed calculation of 
these terms for further work. For the example we discuss later, we parametrically estimated all the loop contributions and chose a point in the parameter space where we can neglect the loops. Nevertheless, we need to point out that we also found region in the parameter space where these loops corrections must be included and can even dominate.

Some comments are in order about the validity of the perturbative analysis. Assume for the moment that $\gamma, \chi \approx 0$ such that only $f_{N L}^{\text {int }}$ is non-zero. Then, in order to be able to compute the 2-pt correlation function of $\delta \chi$ using only the quadratic action like we did, the interaction terms must be small 24]. In our context, it is enough to demand that $V_{, \chi \chi \chi}<H$, or equivalently,

$$
\gamma>2\left(P_{2}^{\zeta}\right)^{1 / 6}\left(\frac{f_{N L}^{\mathrm{int}}}{N_{e}}\right)^{1 / 3},
$$

where we used Eq. (111) and we assumed $\epsilon_{*} \sim \epsilon_{f}$ and $\gamma<1$. This tells us that in order to obtain a large $f_{N L}$ we cannot take the coupling between the two sectors $(\chi$ and $\phi$ ) to be arbitrarily weak. Indeed, the smaller $\gamma$ corresponds to a more strongly interacting scenario for a given $f_{N L}$. Note that this is a computational limit, it might be very interesting to have a strongly coupled hidden sector. We leave this to future investigation. In the case where $\gamma_{, \chi}$ is non zero then one must ensures that the loop factors discussed above are small corrections to the 2-pt function. This can be achieved by taking $\gamma$ sufficiently small.

A multi-field scenario. We illustrate this method of generating non-Gaussian curvature perturbations with a slightly modified version of a $D$-term inflationary scenario arising in IBMs [5]. This IBM scenario uses the inherent extra gauge symmetry of the hidden sector to reduce the amount of fine-tuning required for inflation, and can accommodate Standard Model embeddings with interesting phenomenology. We will find that this wellmotivated scenario will exhibit the rich structure of nonGaussian perturbations which we have discussed above.

The key feature of this inflationary setup is that we have 4 different $\mathrm{D}$-brane stacks with $U(1)_{\mathrm{inf}, 1,2,3}$ gauge theories on their world volumes. There are several chiral multiplets which live at the topological intersections of any two branes with each other and transform in bifundamental of the gauge groups on the two branes. The resulting D-term potential and superpotential are

$$
\begin{aligned}
V_{\mathrm{inf}}^{D}= & \frac{g^{2}}{2}\left(\left|\phi_{+}\right|^{2}-\left|\phi_{-}\right|^{2}-\left|\phi_{N G}\right|^{2}+\cdots-\xi\right)^{2} \\
V_{\text {rest }}^{D}= & \frac{g_{1}^{2}}{2}\left(\left|\phi_{-}\right|^{2}-|S|^{2}+\cdots-\xi_{1}\right)^{2} \\
& +\frac{g_{2}^{2}}{2}\left(|S|^{2}-\left|\phi_{+}\right|^{2}+q\left|S_{N G}\right|^{2}+\cdots-\xi_{2}\right)^{2} \\
& +\frac{g_{3}^{2}}{2}\left(\left|\phi_{N G}\right|^{2}-q\left|S_{N G}\right|^{2}\right)^{2}+\cdots \\
W= & \lambda S \phi_{+} \phi_{-}+\lambda_{N G} S_{N G} \phi_{+} \phi_{N G}
\end{aligned}
$$

where $\cdots$ denotes additional fields which arise at the intersections of these $4 \mathrm{D}$-brane stacks with other branes which are not relevant for us. $V_{\text {inf }}^{D}$ is the D-term potential of the brane which generates inflation, and $q= \pm 1$ is the charge of $S_{N G}$ under $U(1)_{2}$ (in an IBM construction, this sign is determined by the orientation of topological intersection at which the multiplet lives). Note that we have made a particular choice of the sign of the charge for all other fields, but we have left the charge of $S_{N G}$ undetermined because we will find that it has interesting observational consequences. The superpotential will generate F-terms (setting $\phi_{-}=\phi_{N G}=0$ )

$$
V^{F}=\lambda^{2} S^{2} \phi_{+}^{2}+\lambda_{N G}^{2} S_{N G}^{2} \phi_{+}^{2} .
$$

Without any loss of generality, we assume $\xi>0$. As a result, $\phi_{+}$is the waterfall field. $S$ is the inflaton; its vev gives $\phi_{+}$positive mass. $S_{N G}$ is the new field added compared to the set-up in [5] and this will be our field $\chi$. On the D-flat direction, $V_{\text {rest }}^{D}=0$ and $V_{\text {inf }}^{D}=\frac{g^{2}}{2} \xi^{2}$. An inflaton potential is induced from the one-loop ColemanWeinberg potential

$$
\begin{aligned}
V_{\mathrm{inf}}= & \frac{g^{2} \xi^{2}}{2}\left[1+\frac{g^{2}}{16 \pi^{2}} V_{C W}(x)\right], \\
V_{C W}(x)= & \left(x^{2}+1\right)^{2} \ln \left(x^{2}+1\right)-2 x^{4} \ln x^{2} \\
& +\left(x^{2}-1\right)^{2} \ln \left(x^{2}-1\right)-4 \ln 2,
\end{aligned}
$$

where $x=\frac{\lambda^{2} \phi^{2}}{g^{2} \xi}$ and we have neglected $\chi$ contributions to the 1-loop potential (in the regime we will consider, they are small). Using $U(1)$ rotational invariance, we may define

$$
\phi \equiv \operatorname{ReS}, \quad \chi \equiv \operatorname{Re} \mathrm{S}_{\mathrm{NG}}, \quad T \equiv \operatorname{Re} \phi_{+},
$$

and we can set all the imaginary parts to zero. The hidden sector field $\chi$ has a quartic coupling given by $V_{\text {hid }}=\frac{\nu^{2}}{4} \chi^{4}=\frac{g_{2}^{2}+g_{3}^{2}}{2} \chi^{4}$. Finally the mass of the tachyonic field depend on the quadratic sum of two fields

$$
m_{T}^{2}=-g^{2} \xi+\lambda^{2} \phi^{2}+\left(\lambda_{N G}^{2}-q g_{2}^{2}\right) \chi^{2} .
$$

Inflation ends when the inflaton reaches the critical value $\phi_{c}^{2}=\frac{g^{2} \xi}{\lambda^{2}}+\frac{\left(q g_{2}^{2}-\lambda_{N G}^{2}\right) \chi^{2}}{\lambda^{2}}$ which gives

$$
\gamma=\left.\frac{\partial \phi_{c}}{\partial \chi}\right|_{\phi_{c}}=\frac{q g_{2}^{2}-\lambda_{N G}^{2}}{\lambda^{2}} \frac{\bar{\chi}}{\phi_{c}} .
$$

We denote the stochastic part of the field $\chi$ by $\bar{\chi}$, which is a random variable with a mean of zero. The quantum perturbations of $\chi$ grows to be of order $H$ at horizon exit. At this point, one can assume a semi-classical treatment where the field $\chi$ is undergoing a random walk with steps of order $H$ for each interval of time $1 / H$. The FokkerPlanck equation then gives the non-Gaussian probability distribution for $\bar{\chi}$. We thus find $\bar{\chi}=\sigma H / \sqrt{\nu}$, where a typical range is $-0.6<\sigma<0.6[7]$. Note that $\gamma$ depends 
on this stochastic parameter and this is one of the main difference from [7].

Analysis. At this point the system has been reduced to D-term inflation. In our analysis we will take the limit $\frac{\xi}{\lambda^{2}} \gg \frac{N_{e} M_{p}^{2}}{2 \pi^{2}}$ and assume that $\gamma \ll 1$ as well as $\phi_{c}^{2} \sim \frac{g^{2} \xi}{\lambda^{2}}$. In this limit, $\phi_{*}^{2} \sim \phi_{f}^{2} \sim \frac{g^{2} \xi}{\lambda^{2}}$ (or equivalently $\epsilon_{*} \sim \epsilon_{f}$ ), and the inflaton does not move much during inflation. We must go to this limit in order to realize significant non-Gaussianity, because the perturbations to $\delta N$ can arise both from fluctuations at the start of inflation and from the end. The initial fluctuations (in our model) are Gaussian, so significant non-Gaussianity can only arise if the non-Gaussian perturbations at the end of inflation are of about the same scale. This also means that the spectral index will be close to one. In this regime,the 2-pt. normalization is

$$
P_{\zeta} \sim 2.28 \times 10^{-9} \sim \frac{4 \pi^{2}}{6} \frac{\xi^{3}}{(2 \ln (2))^{2} \lambda^{2} M_{p}^{6}} .
$$

The local and intrinsic contributions to $f_{N L}$ are

$$
\begin{aligned}
f_{N L}^{\text {int }} & \sim \frac{5 \ln (2)}{2 \pi^{2}} \frac{\nu^{2} N_{e} \kappa^{6} \bar{\chi}^{4}\left(q g_{2}^{2}-\lambda_{N G}^{2}\right)^{3} M_{p}^{4}}{\lambda^{2} \xi^{4} g^{4}} \\
f_{N L}^{\text {loc }} & \sim-\frac{5 \ln (2)}{12 \pi^{2}} \frac{\bar{\chi}^{2}\left(q g_{2}^{2}-\lambda_{N G}^{2}\right)^{3} \kappa^{4} M_{p}^{2}}{\lambda^{2} \xi^{2}}
\end{aligned}
$$

The sign of $f_{N L}$ is determined by the sign of $q g_{2}^{2}-\lambda_{N G}^{2}$. Note that this dependence arises from the way these couplings affect the mass of the waterfall field; the Yukawa coupling adds a positive mass contribution and delays the end of inflation, while the gauge coupling term adds a mass contribution whose sign depends on the charge $q$, and can either delay or hasten the end of inflation. In particular, if $q=+1$ and $g_{2}^{2}>\lambda_{N G}^{2}$, then $q g_{2}^{2}-\lambda_{N G}^{2}>0$. If this model arises from the type of IBM construction described in [5], then one would indeed expect $g_{2}^{2}>\lambda_{N G}^{2}$, because Yukawa couplings are exponentially suppressed (in Type IIA string theory, they arise from worldsheet instatons). On the other hand, if $q=-1$, then we can set $\lambda_{N G}=0$, because the Yukawa term $\lambda_{N G} S_{N G} \phi_{+} \phi_{N G}$ is not classically gauge-invariant (both $\phi_{+}$and $S_{N G}$ would have charge -1 under $\left.U(1)_{2}\right)$. We then find that $q g_{2}^{2}<0$. We thus directly see how the sign of the charge of the new scalar determines the sign of the various contributions to $f_{N L}$ in this model.

There are a few consistency conditions one must satisfy; we take $\eta_{\chi} \lesssim 0.01$ so that the non-Gaussian perturbations in $\chi$ do not decay significantly between horizoncrossing and the end of inflation. For perturbative control we must satisfy Eq. (18). As a toy example, we take $\xi \sim 2.9 \times 10^{-6} M_{p l}^{2}, \lambda \sim 2 \times 10^{-4}, q g_{2}^{2}-\lambda_{N G}^{2} \sim-0.17$, $\nu \sim 0.05, g^{2} \sim 10^{-3}$ and $\bar{\chi} \sim 0.12 H / \sqrt{\nu}$. We then satisfy the above consistency conditions and generate $\gamma \sim-0.5$ and

$$
\begin{aligned}
f_{N L}^{\text {int }} & \sim-8, & n_{s} & \sim 1.002, \\
f_{N L}^{\text {loc }} & \sim 45, & G \mu & \sim 7 \times 10^{-7} .
\end{aligned}
$$

where we included the cosmic strings produced in such models (with $G \mu=\xi / 4$ ). For this point in parameterspace, one can check that the loop corrections Eq. (15, 16) scales as $N_{e} \nu^{2}$ and $H^{2} / \chi^{2}$ respectively and for the parameters chosen above, they are small. But clearly for other points in parameter space one or both of these expansions parameters could be significant, in which case one would have to compute higher-order terms as well.

As is typical in D-term inflation, this model predicts cosmic strings. When their contribution to the power spectrum is correctly accounted for, $n_{s} \sim 1$ can be completely consistent with WMAP data [11. One expects that larger values of $\xi$ can lead to smaller values of $n_{s}$ (though if the resulting cosmic string tension exceeds observational bounds, the strings must be unstable). In this case, large values of $f_{N L}$ would require larger values of $q g_{2}^{2}-\lambda_{N G}^{2}$. We can also find points in the parameter space with large $f_{N L}$ where $G \mu$ is smaller (and satisfy the bound of [25]) but in this case, the WMAP bound on $n_{s}$ while not excluding our model, does not favor it. Also, we find that the smaller the cosmic string tension the harder it becomes to satisfy all the consistency conditions and yet get large $f_{N L}$. We have assumed that the vev of the inflaton is sub-planckian. This implies that the energy density in gravity waves will be unobservably small. Significant gravity waves can be generated if the inflaton traverses super-Planckian scales, but in that limit the effective field theory is no longer valid. Finally, we should mention that a different mechanism for generating NG from the tachyon was presented in [26].

Discussion. We have illustrated a simple model for generating primordial non-Gaussian curvature perturbations. This model can arise easily from intersecting brane models and allows a large $f_{N L}$ with either sign which one expects to settle soon from the results of WMAP and Planck. The shape of the non-Gaussianity is local. One can fit the CMB data with large non-Gaussianity and a flat spectrum once the contribution from cosmic strings is included correctly. Without the cosmic strings, the current bound on $n_{s}$ implies this model is not favored by the data but not excluded. The fact that the tachyon mass depends on additional fields is a feature shared by many string theory models of inflation [27].

In this general and well-motivated effective field theory model, we have found a relatively complicated structure to the non-Gaussian contributions, with contributions from both intrinsic and non-linear terms, and possible loop-contributions as well. The non-linear contributions are usually dominant. We have found that the sign of $f_{N L}$ depends largely on the hidden-sector charge of the extra field, and on the relative importance of the gauge 
and Yukawa sectors.

Another model of D-term inflation in string theory was recently reanalysed in 28] (and effects from entropy modes were discussed in 29]) and it will be interesting to study whether NG can also be generated in their model. An important difference in the D3/D7 model compared to the IBM model we presented is the fact that in the former, the Yukawa coupling and gauge coupling are the same. Therefore to achieve the $n_{s} \sim 1$ regime, they need a very small gauge coupling (of order $10^{-4}$ ) while in our case it can be larger. Of course, the D3/D7 model contains a full string theory description with backreaction and moduli stabilization effects included. It would be interesting to see if NG of the type presented in this work still survives in a more concrete model from string theory.

We are grateful to R. Brandenberger, K. Dasgupta, B Garbrecht, M. Kaplinghat, E. Komatsu, R. Holman, A. Linde, S. Shandera and M. Wyman for useful discussions, and to the KITP for its hospitality. We are particularly thankful to D. Lyth for pointing out to us the importance of the local contribution to the NG. This work is supported in part by DOE Grant DE-FG02-95ER40917, NSF Grants No. PHY-0239817, PHY-0653656, PHY0551164 and PHY-0505757.

* Electronic address: dutta@physics.tamu.edu

$\dagger$ Electronic address: lleblond@physics.tamu.edu

¥ Electronic address: kumarj@uci.edu

[1] E. Komatsu et al. [WMAP Collaboration], arXiv:0803.0547 [astro-ph].

[2] A. P. S. Yadav and B. D. Wandelt, arXiv:0712.1148 [astro-ph].

[3] P. Creminelli, L. Senatore, M. Zaldarriaga and M. Tegmark, JCAP 0703, 005 (2007) arXiv:astro-ph/0610600.

[4] J. M. Maldacena, JHEP 0305, 013 (2003) arXiv:astro-ph/0210603; V. Acquaviva, N. Bartolo, S. Matarrese and A. Riotto, Nucl. Phys. B 667, 119 (2003) arXiv:astro-ph/0209156.

[5] B. Dutta, J. Kumar and L. Leblond, JHEP 0707, 045 (2007) arXiv:hep-th/0703278.

[6] P. Binetruy and G. R. Dvali, Phys. Lett. B 388, 241 (1996) arXiv:hep-ph/9606342; E. Halyo, Phys. Lett. B 387 (1996) 43 arXiv:hep-ph/9606423.

[7] F. Bernardeau and T. Brunier, Phys. Rev. D 76, 043526 (2007) arXiv:0705.2501 [hep-ph]].

[8] N. Bartolo, S. Matarrese and A. Riotto, Phys. Rev. D 65, 103505 (2002) arXiv:hep-ph/0112261; F. Bernardeau and J. P. Uzan, Phys. Rev. D 66, 103506 (2002) arXiv:hep-ph/0207295; F. Bernardeau and J. P. Uzan, Phys. Rev. D 67, 121301 (2003) arXiv:astro-ph/0209330.

[9] L. Alabidi and D. Lyth, JCAP 0608, 006 (2006) arXiv:astro-ph/0604569; L. Alabidi, JCAP 0610, 015 (2006) arXiv:astro-ph/0604611; M. Sasaki, arXiv:0805.0974 [astro-ph].

[10] G. Dvali, A. Gruzinov and M. Zaldarriaga, Phys. Rev. D
69, 023505 (2004) arXiv:astro-ph/0303591; G. Dvali, A. Gruzinov and M. Zaldarriaga, Phys. Rev. D 69, 083505 (2004) arXiv:astro-ph/0305548.

[11] R. A. Battye, B. Garbrecht and A. Moss, JCAP 0609, 007 (2006) arXiv:astro-ph/0607339. N. Bevis, M. Hindmarsh, M. Kunz and J. Urrestilla, Phys. Rev. Lett. 100, 021301 (2008) arXiv:astro-ph/0702223.

[12] M. Sasaki and E. D. Stewart, Prog. Theor. Phys. 95, 71 (1996) arXiv:astro-ph/9507001.

[13] A. D. Linde and V. F. Mukhanov, Phys. Rev. D 56, 535 (1997) arXiv:astro-ph/9610219; D. H. Lyth and D. Wands, Phys. Lett. B 524, 5 (2002) arXiv:hep-ph/0110002.

[14] E. I. Buchbinder, J. Khoury and B. A. Ovrut, arXiv:0710.5172 [hep-th]; J. L. Lehners and P. J. Steinhardt, Phys. Rev. D 77, 063533 (2008) arXiv:0712.3779 [hep-th]]; J. L. Lehners and P. J. Steinhardt, arXiv:0804.1293 [hep-th].

[15] D. H. Lyth, K. A. Malik and M. Sasaki, JCAP 0505, 004 (2005) arXiv:astro-ph/0411220; D. H. Lyth and Y. Rodriguez, Phys. Rev. Lett. 95, 121302 (2005) arXiv:astro-ph/0504045.

[16] T. Falk, R. Rangarajan and M. Srednicki, Astrophys. J. 403 (1993) L1 arXiv:astro-ph/9208001.

[17] M. Zaldarriaga, Phys. Rev. D 69 (2004) 043508 arXiv:astro-ph/0306006; D. Seery, K. A. Malik and D. H. Lyth, JCAP 0803 (2008) 014 arXiv:0802.0588 [astro-ph]].

[18] D. Babich, P. Creminelli and M. Zaldarriaga, JCAP 0408, 009 (2004) arXiv:astro-ph/0405356.

[19] D. Seery and J. E. Lidsey, JCAP 0506 (2005) 003 arXiv:astro-ph/0503692. X. Chen, M. x. Huang, S. Kachru and G. Shiu, JCAP 0701, 002 (2007) arXiv:hep-th/0605045.

[20] N. Barnaby, T. Biswas and J. M. Cline, JHEP 0704, 056 (2007) arXiv:hep-th/0612230. N. Barnaby and J. M. Cline, JCAP 0707, 017 (2007) arXiv:0704.3426 [hep-th]]. N. Barnaby and J. M. Cline, arXiv:0802.3218 [hep-th].

[21] D. H. Lyth, JCAP 0712, 016 (2007) arXiv:0707.0361 [astro-ph]].

[22] M. Crocce and R. Scoccimarro, Phys. Rev. D 73, 063519 (2006) arXiv:astro-ph/0509418. C. T. Byrnes, K. Koyama, M. Sasaki and D. Wands, JCAP 0711, 027 (2007) arXiv:0705.4096 [hep-th]]. D. Seery, JCAP 0802, 006 (2008) arXiv:0707.3378 [astro-ph]].

[23] I. Zaballa, Y. Rodriguez and D. H. Lyth, JCAP 0606, 013 (2006) arXiv:astro-ph/0603534.

[24] L. Leblond and S. Shandera, arXiv:0802.2290 [hep-th].

[25] L. Pogosian, S. H. H. Tye, I. Wasserman and M. Wyman, Phys. Rev. D 68, 023506 (2003) [Erratum-ibid. D 73, 089904 (2006)] arXiv:hep-th/0304188.

[26] K. Enqvist, A. Jokinen, A. Mazumdar, T. Multamaki and A. Vaihkonen, JCAP 0503, 010 (2005) arXiv:hep-ph/0501076.

[27] D. H. Lyth and A. Riotto, Phys. Rev. Lett. 97, 121301 (2006) arXiv:astro-ph/0607326; L. Leblond and S. Shandera, JCAP 0701, 009 (2007) arXiv:hep-th/0610321.

[28] M. Haack, R. Kallosh, A. Krause, A. Linde, D. Lust and M. Zagermann, arXiv:0804.3961 [hep-th].

[29] R. H. Brandenberger, K. Dasgupta and A. C. Davis, arXiv:0801.3674 [hep-th]. 